\title{
Evaluation of Drought Tolerance in Rapeseed Genotypes under Non Stress and Drought Stress Conditions
}

\author{
Amir Hossein SHIRANI RAD ${ }^{1 *}$, Abouzar ABBASIAN ${ }^{2}$ \\ ${ }^{1}$ Seed and Plant Improvement Institute, Karaj, Iran; Shirani.Rad@gmail.com ( ${ }^{*}$ corresponding author) \\ ${ }^{2}$ Young Researchers Club, Shahr-e-Qods Branch, Islamic Azad University, Tehran, Iran
}

\begin{abstract}
Drought is a wide spread problem seriously influencing rapeseed (Brassica napus L.) production, mostly in dryland regions. This study was conducted to determine drought tolerance genotypes with superiority in different stressed environments. Twenty three rapeseed genotypes were tested in a split plot design based on randomized complete block design (RCBD) with four replications in two years (2008- 2009 and 2009-2010) at Seed and Plant Improvement Institute of Karaj, Iran. Seven drought resistance indices include susceptible stress index (SSI), tolerance index (TOL), stress mean productivity (MP), geometric mean productivity (GMP), stress tolerance index (STI), yield index (YI) and yield stability index (YSI) were applied on the basis of seed yield in non stress and drought stress conditions. Based on different drought indices, genotypes 'Modena', 'Geronimo', 'Elite', 'Syn-4' and 'SLM046' had the best rank with low standard deviation. The results indicated that they have stable yield performance. Bi-plot display and cluster analysis cleared superiority of these genotypes in both years. The synthetic derived cultivars could perform well across all environments with better agronomic performance. Results showed MP, GMP and YI indices were more effective in identifying high yielding cultivars in diverse water scarcity.
\end{abstract}

Keywords: drought stress, oil yield, rapeseed, seed yield, sensitivity, tolerance index

\section{Introduction}

Rapeseed is an important oil seed crop in the agricultural systems of many arid and semiarid areas where its yield is often restricted by water deficit and high temperatures during the reproductive growth. Oil rapeseed has $61 \%$ oleic acid and $8.8 \%$ linoleic acid that in comparison with another oil seeds have better quality (Afridi et al., 2002). Seed yield can be primarily limited even by the relatively short period of soil moisture shortage during the reproductive development (Ahmadi and Bahrani, 2009). The effect of water stress on crop is a function of reduction genotype, intensity and duration of stress, weather conditions and developmental stages of rapeseed (Robertson and Holland, 2004). Water stress and high temperature can reduce crop yield by affecting both source and sink for assimilates (Mendham and Salsbury, 1995).

Because of water deficit in most arid regions, resistance of crop plants against drought has always been of great importance and has taken into account as one of the breeding factors (Talebi, 2009). A long term drought stress effects on plant metabolic reactions associate with plant growth stage, water storage capacity of soil and physiological aspects of plant. Drought tolerance in crop plants is different from wild plants. In case crop plant that encounters with severe water deficit, they die or seriously lose yield while in wild plants, they survive under this conditions but yield losses is not taken into consideration (Khayatnezhad et al.,
2010). Achieving a genetic increase in yield under these environments has been recognized to be a difficult challenge for plant breeders while progress in yield grain has been much higher in favorable environments (Richards et al., 2002). Thus, drought indices which provide a measure of drought based on yield loss under drought conditions in comparison to normal conditions have been used for screening drought tolerant genotypes (Mitra, 2001).

To evaluate response of plant genotypes to drought stress, some selection indices based on a mathematical relation between stress and optimum conditions have been proposed (Clarke et al., 1992; Fernandez, 1992; Sio-Se Mardeh et al., 2006; Shirani Rad and Abbasian, 2011). Rosielle and Hamblin (1981) defined stress tolerance (TOL) as the differences in yield between the stress (Ys) and non-stress ( $\mathrm{Yp}$ ) environments and mean productivity (MP) as the average yield of Ys and Yp. Fischer and Maurer (1978) proposed a stress susceptibility index (SSI) of the cultivar. Fernandez (1992) defined a new advanced index (STI = stress tolerance index), which can be used to identify genotypes that produce high yield under both stress and non-stress conditions. Geometric mean productivity (GMP) and stress tolerance index (STI) (Fernandez, 1992) have been employed under various conditions. Fischer and Maurer (1978) explained that genotypes with an SSI of less than a unit are drought resistant, since their yield reduction in drought conditions is smaller than the mean yield reduction of all genotypes (Bruckner and Frohberg, 
1987). Other yield based estimates of drought resistance are harmonic mean (HM) (Dehdari, 2003; Yousefi, 2004), yield index (YI) (Gavuzzi et al., 1997), yield stability index (YSI) (Bouslama and Schapaugh, 1984) and \% reduction (Choukan et al., 2006). Sio-Se Mardeh et al. (2006) reported that under moderate stress, MP, GMP and STI were more effective in identifying high yielding cultivars in both drought-stressed and irrigated conditions (group A cultivars). Under severe stress, none of the indices used were able to identify group A cultivars, although regression coefficient (b) and SSI were found to be more useful in discriminating resistant cultivars. So, the effectiveness of selection indices in differentiating resistant cultivars varies with the stress severity.

The objective of this experiment was to determine best cultivar based on influences of water stress at stem elongation stage on yield and oil yield of rapeseed in Karaj, Iran, a main rapeseed growing area in Iran. There is a high potential for expansion of rapeseed cultivation in these regions as a promising alternative crop for diversification and economical use of land and water resources. The suitability of indicators seems to depend on the timing and severity of stress in drought prone environments. The objective of this study was to test this hypothesis in order to identify the most suitable indices/cultivars for each environment.

\section{Materials and methods}

This study was carried out at the experimental farm of Seed and Plant Improvement Institute, Karaj, Iran (latitude $35^{\circ} 55^{\prime} \mathrm{N}$, longitude $50^{\circ} 54^{\prime} \mathrm{E}$, elevation 1,313 $\mathrm{m}$ above mean sea level) during 2008-2010. This region has a semi-arid climate $(354 \mathrm{~mm}$ annual rainfall). The soil of the experimental site is a clay loam, with montmorillionite clay mineral, low in nitrogen $(0.07-0.08 \%)$, low in organic matter $(0.54-0.62 \%)$, and alkaline in reaction, with a $\mathrm{pH}$ of 7.8 and $\mathrm{EC}=0.69 \mathrm{dS} \mathrm{\textrm {m } ^ { - 1 }}$. The soil texture is sandy loam, with $12 \%$ of neutralizing substances. The experimental design was split plot based on randomized complete block design (RCBD) with four replications. Two irrigation levels consisting of irrigation after $80 \mathrm{~mm}$ evaporation from class "A" pan as control (irrigation during full season) and no irrigation from stem elongation stage were applied in main plots and subplots which consisted of split application of winter rapeseed cultivars at 23 levels ('SW0756', 'Modena', 'Geronimo, 'Elite', 'Opera', 'ARC-4', 'ARG-91004', 'ARC-5', 'ARC-2', 'Digger', 'Adder,' 'Milena, 'RG9908, 'Dexter,' 'Alice,' 'Olara, 'Ebonite', 'Syn-4,' 'Zarfam,' 'SLM046, 'Okapi', 'Orient' and 'Elvice') based on their reputed differences in yield performance under irrigated and non-irrigated conditions and main cultivars of Karaj region.

Individual plot consisted of 6 rows, $6 \mathrm{~m}$ long and spaced $30 \mathrm{~cm}$ apart using a seeding rate of $7 \mathrm{~kg} \mathrm{ha}^{-1}$. The experimental fields were mould-board ploughed and seedbed preparation consisted of two passes with a tandem disk. Seeds were planted 1 to $1.5 \mathrm{~cm}$ deep at a rate of 100 seeds $\mathrm{m}^{-2}$ on 5 October 2008 and 2009. For all treatments, $\mathrm{N}: \mathrm{P}: \mathrm{K}$ fertilizers were applied at rates of $150: 60: 50 \mathrm{~kg}$ ha $^{-1}$, respectively. All of $\mathrm{P}, \mathrm{K}$ fertilizer and one-third of $\mathrm{N}$ fertilizer were incorporated and added to soil pre-sowing. Other two-third of $\mathrm{N}$ fertilizer was split equally at the beginning of stem elongation and flowering stages. Weeds were controlled by application of haloxyfop- R-methyl ester (Gallant Super, $10 \%$ EC) at $0.6 \mathrm{~L} \mathrm{ha}^{-1}$. Broadleaf weeds were also hand weeded during the season. Final harvests were carried out on 10 June 2009 and 25 June 2010.

The seed yield was measured by harvesting $4.8 \mathrm{~m}^{2}$ of the central part of each plot at crop maturity. Oil content was determined by the nuclear magnetic resonance (NMR). Oil yield was obtained multiplying seed yield by oil content. Drought resistance indices were calculated using the following relationships:

$$
\text { (1) } S S I=\frac{1-\left(Y_{s} / Y_{p}\right)}{1-(\overline{Y s} / \overline{Y p})} \text { (Fischer and Maurer, 1978) }
$$

where Ys is the yield of cultivar under stress, Yp the yield of cultivar under irrigated condition, $\overline{Y s}$ and $\overline{Y p}$ are the mean yields of all cultivars under stress and non-stress conditions, respectively, and $1-\left(\overline{Y_{s}} / \overline{Y_{p}}\right)$ is the stress intensity. The irrigated experiment was considered to be nonstress conditions in order to have a better estimation of optimum environment.

$$
\begin{aligned}
& \text { (2) } M P=\frac{Y p+Y s}{2} \text { (Hossain et al., 1990) } \\
& \text { (3) } T O L=Y p-Y_{s} \quad(\text { Hossain } \text { et al., 1990) } \\
& \text { (4) } S T I=\frac{Y p \times Y s}{(\overline{Y p})^{2}}(\text { Fernandez, 1992) } \\
& \text { (5) } G M P=\sqrt{Y_{p} \times Y_{s}} \quad \text { Fernandez, 1992) } \\
& \text { (6) Yield index (YI) }=\frac{Y s}{\overline{Y s}} \text { (Gavuzzi et al., 1997) } \\
& \text { (7) Yield stability index (YSI) }=\frac{Y_{s}}{Y_{p}} \text { (Bouslama and Schapaugh, 1984) } \\
& \text { (8) } \% \text { Reduction }=\frac{Y p \quad Y s}{Y p} \times 100 \text { (Choukan et al., 2006) }
\end{aligned}
$$

The data were analyzed using SAS software (SAS System, 1996) for analysis of variance and cluster analysis of genotypes based on Euclidean distance, and Duncan's multiple range test $(\mathrm{p} \leq 0.05)$ was employed for the mean comparisons. The biplot display was also used to identify tolerant and high yielding genotypes using StatGraphics software, based on principal component analysis.

\section{Results and discussion}

Resistance indices were calculated on the basis of seed and oil yield of cultivars (Tab. 1, 2, 3 and 4). Selection based on a combination of indices may provide a more useful criterion for improving drought resistance of rapeseed but study of correlation coefficients is useful in finding the 
166

degree of overall linear association between any two attributes. Accordingly, high levels indicators STI, MP, GMP, YI and YSI values and low index of TOL and SSI indicator of resistance to stress conditions were figured. Fernandez (1992) classified plants according to their performance in stressful and stress free environments to four groups: genotypes with similar good performance in both environments (group A), genotypes with good performance only in non-stress environments (group B) or stressful environments (group C), and genotypes with weak performance in both environments (group D) (Fig. 1).

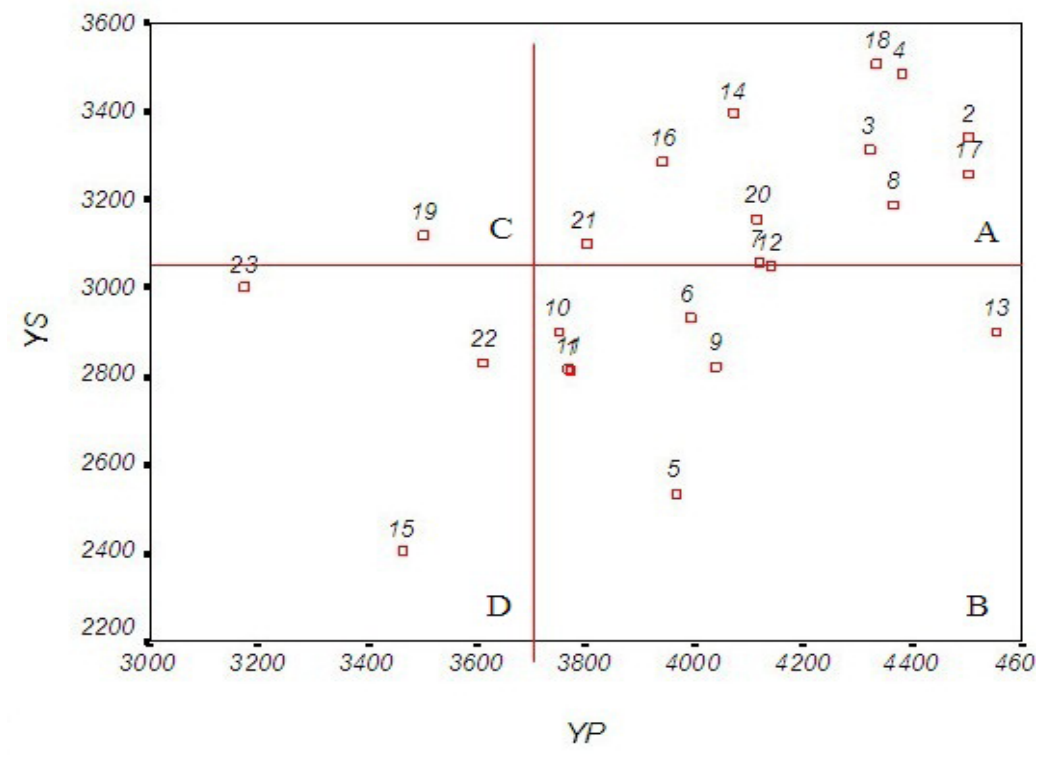

Fig. 1. The relationship between seed yield produced under non stress and drought stress environments in mean of two years

Tab. 1. Resistance indices of 23 rapeseed genotypes under stress and non-stress environments for seed yield in 2008-2009

\begin{tabular}{|c|c|c|c|c|c|c|c|c|c|c|}
\hline Cultivar & $\mathrm{Yp}\left(\mathrm{kg} \cdot \mathrm{ha}^{-1}\right)$ & Ys $\left(\mathrm{kg} \cdot \mathrm{ha}^{-1}\right)$ & SSI & TOL & MP & GMP & STI & YI & YSI & Reduction (\%) \\
\hline 'SW0756 & $3636 a-f$ & $2325 \mathrm{klm}$ & 1.13 & 1311 & 2980.5 & 2907.5 & 0.63 & 0.93 & 0.64 & 36.05 \\
\hline 'Modena' & $4315 \mathrm{a}$ & $2583 \mathrm{~h}-\mathrm{m}$ & 1.26 & 1732 & 3449 & 3338.5 & 0.83 & 1.03 & 0.59 & 40.13 \\
\hline 'Geronimo' & $4140 \mathrm{ab}$ & $2428 j-m$ & 1.3 & 1712 & 3284 & 3170.5 & 0.75 & 0.97 & 0.58 & 41.35 \\
\hline 'Elite' & $3836 \mathrm{a}-\mathrm{d}$ & $2907 \mathrm{f}-\mathrm{m}$ & 0.76 & 929 & 3371.5 & 3339.349 & 0.83 & 1.16 & 0.76 & 24.21 \\
\hline 'Opera’ & $3533 \mathrm{a}-\mathrm{g}$ & $2169 \mathrm{~m}$ & 1.21 & 1364 & 2851 & 2768.2 & 0.57 & 0.87 & 0.61 & 38.6 \\
\hline 'ARC-4' & $3383 \mathrm{~b}-\mathrm{h}$ & $2163 \mathrm{~m}$ & 1.13 & 1214 & 2776 & 2708.8 & 0.55 & 0.87 & 0.64 & 35.88 \\
\hline 'ARG-91004' & $3837 \mathrm{a}-\mathrm{d}$ & $2312 \mathrm{klm}$ & 1.25 & 1525 & 3074.5 & 2978.4 & 0.66 & 0.92 & 0.6 & 39.74 \\
\hline 'ARC-5' & $3835 \mathrm{a}-\mathrm{d}$ & $2290 \mathrm{klm}$ & 1.27 & 1545 & 3062.5 & 2963.5 & 0.65 & 0.91 & 0.59 & 40.29 \\
\hline 'ARC-2' & 3681 a-f & $2336 \mathrm{klm}$ & 1.15 & 1345 & 3008.5 & 2932.4 & 0.64 & 0.93 & 0.63 & 36.54 \\
\hline 'Digger' & $3225 \mathrm{~d}-\mathrm{j}$ & $2261 \mathrm{~lm}$ & 0.94 & 964 & 2743 & 2700.319 & 0.54 & 1.01 & 0.7 & 29.89 \\
\hline 'Adder' & 3838 a-d & $2336 \mathrm{klm}$ & 1.23 & 1502 & 3087 & 2994.3 & 0.67 & 0.93 & 0.6 & 39.13 \\
\hline 'Milena' & 3725 a-e & $2348 \mathrm{klm}$ & 1.16 & 1377 & 3036.5 & 2957.4 & 0.65 & 0.94 & 0.63 & 36.96 \\
\hline 'RG-9908' & $4110 \mathrm{abc}$ & $2481 \mathrm{j}-\mathrm{m}$ & 1.25 & 1629 & 3295.5 & 3193.3 & 0.76 & 0.99 & 0.6 & 39.63 \\
\hline 'Dexter' & 3669 a-f & $2511 \mathrm{i}-\mathrm{m}$ & 0.99 & 1158 & 3090 & 3035.3 & 0.69 & 1.01 & 0.68 & 31.56 \\
\hline 'Alice' & 3627 a-f & $2504 \mathrm{i}-\mathrm{m}$ & 0.97 & 1123 & 3065.5 & 3013.6 & 0.68 & 1.002 & 0.69 & 30.96 \\
\hline 'Olara' & 3658 a-f & 2960 e-m & 0.6 & 698 & 3309 & 3290.5 & 0.81 & 1.18 & 0.81 & 19.08 \\
\hline 'Ebonite' & $4308 \mathrm{a}$ & 3013 d-1 & 0.94 & 1295 & 3660.5 & 3602.8 & 0.97 & 1.2 & 0.69 & 30.06 \\
\hline 'Syn-4' & 3834 a-d & 3031 d-1 & 0.66 & 803 & 3432.5 & 3408.9 & 0.86 & 1.21 & 0.79 & 20.94 \\
\hline 'Zarfam' & $3108 \mathrm{~d}-\mathrm{k}$ & $2583 \mathrm{~h}-\mathrm{m}$ & 0.53 & 525 & 2845.5 & 2833.4 & 0.59 & 1.03 & 0.83 & 16.89 \\
\hline 'SLM046' & 3771 a-e & $2486 \mathrm{i}-\mathrm{m}$ & 1.07 & 1285 & 3128.5 & 3061.8 & 0.69 & 0.99 & 0.66 & 34.07 \\
\hline 'Okapi' & $3300 \mathrm{c}-\mathrm{i}$ & 2600 h-m & 0.67 & 700 & 2950 & 2929.2 & 0.64 & 1.04 & 0.79 & 21.21 \\
\hline 'Orient' & $3114 \mathrm{~d}-\mathrm{k}$ & $2329 \mathrm{klm}$ & 0.79 & 785 & 2721.5 & 2693 & 0.54 & 0.93 & 0.75 & 25.2 \\
\hline 'Elvice' & $2750 \mathrm{~g}-\mathrm{m}$ & $2500 \mathrm{klm}$ & 0.28 & 250 & 2625 & 2622 & 0.51 & 1.0007 & 0.91 & 9.09 \\
\hline
\end{tabular}

Ys: yield of cultivar under stress, Yp: yield of cultivar under irrigated condition, SSI: stress susceptibility index, TOL: Tolerance, MP: mean productivity, GMP: Geometric mean productivity, STI: stress tolerance index, YI: yield index, YSI: yield stability index 
Tab. 2. Resistance indices of 23 rapeseed genotypes under stress and non-stress environments for seed yield in 2009-2010

\begin{tabular}{|c|c|c|c|c|c|c|c|c|c|c|}
\hline Cultivar & Yp $\left(\mathrm{kg} \cdot \mathrm{ha}^{-1}\right)$ & Ys $\left(\mathrm{kg} \cdot \mathrm{ha}^{-1}\right)$ & SSI & TOL & MP & GMP & STI & YI & YSI & Reduction (\%) \\
\hline 'SW0756' & $3913 \mathrm{f}-\mathrm{p}$ & $3300 \mathrm{pq}$ & 0.91 & 613 & 3606.5 & 3593.5 & 0.69 & 0.92 & 0.84 & 15.66 \\
\hline 'Modena' & 4694 a-e & 4100 e-n & 0.74 & 594 & 4397 & 4386.958 & 1.01 & 1.14 & 0.87 & 12.65 \\
\hline 'Geronimo' & $4500 a-h$ & $4200 \mathrm{~d}-\mathrm{m}$ & 0.39 & 300 & 4350 & 4347.4 & 0.99 & 1.16 & 0.93 & 6.66 \\
\hline 'Elite' & $4923 \mathrm{ab}$ & 4058 e-n & 1.021 & 865 & 4490.5 & 4469.6 & 1.05 & 1.12 & 0.82 & 17.57 \\
\hline 'Opera' & $4400 a-j$ & $2900 \mathrm{q}$ & 1.98 & 1500 & 3650 & 3572.1 & 0.67 & 0.8 & 0.66 & 34.1 \\
\hline ARC-4' & $4597 \mathrm{a}-\mathrm{f}$ & $3698 \mathrm{k}-\mathrm{p}$ & 1.14 & 899 & 4147.5 & 4123.1 & 0.89 & 1.02 & 0.8 & 19.56 \\
\hline 'ARG-91004' & $4400 a-j$ & 3800 i-p & 0.79 & 600 & 4100 & 4089 & 0.88 & 1.05 & 0.86 & 13.64 \\
\hline 'ARC-5' & $4894 \mathrm{abc}$ & 4088 e-n & 0.96 & 806 & 4491 & 4472.9 & 1.05 & 1.13 & 0.83 & 16.47 \\
\hline 'ARC-2' & $4400 a-j$ & 3303 opq & 1.45 & 1097 & 3851.5 & 3812.2 & 0.76 & 0.92 & 0.75 & 24.93 \\
\hline 'Digger' & 4277 b-l & $3543 \mathrm{~m}-\mathrm{q}$ & 0.99 & 734 & 3910 & 3892.7 & 0.79 & 0.98 & 0.83 & 17.16 \\
\hline 'Adder' & $3700 \mathrm{k}-\mathrm{p}$ & $3300 \mathrm{pq}$ & 0.63 & 400 & 3500 & 3494.281 & 0.64 & 0.92 & 0.89 & 10.81 \\
\hline 'Milena' & $4554 \mathrm{a}-\mathrm{g}$ & $3746 j-p$ & 1.03 & 808 & 4150 & 4130.3 & 0.89 & 1.039 & 0.82 & 17.74 \\
\hline 'RG-9908' & $5000 \mathrm{a}$ & 3318 opq & 1.95 & 1682 & 4159 & 4073.1 & 0.87 & 0.92 & 0.66 & 33.64 \\
\hline 'Dexter' & $4475 a-i$ & 4277 b-l & 0.25 & 198 & 4376 & 4374.9 & 1.01 & 1.19 & 0.95 & 4.42 \\
\hline 'Alice' & $3300 \mathrm{pq}$ & $2300 \mathrm{r}$ & 1.76 & 1000 & 2800 & 2755 & 0.4 & 0.64 & 0.69 & 30.3 \\
\hline 'Olara' & $4225 \mathrm{c}-\mathrm{m}$ & $36131-p$ & 0.84 & 612 & 3919 & 3907 & 0.8 & 1 & 0.85 & 14.48 \\
\hline 'Ebonite' & 4700 a-e & $3500 \mathrm{n}-\mathrm{q}$ & 1.49 & 1200 & 4100 & 4055.9 & 0.87 & 0.97 & 0.74 & 25.53 \\
\hline 'Syn-4' & $4838 \mathrm{a}-\mathrm{d}$ & $3986 \mathrm{f}-\mathrm{o}$ & 1.02 & 852 & 4412 & 4391.4 & 1.02 & 1.1 & 0.82 & 17.61 \\
\hline 'Zarfam' & 3900 g-p & $3650 \mathrm{k}-\mathrm{p}$ & 0.37 & 250 & 3775 & 3772.9 & 0.75 & 1.01 & 0.94 & 6.41 \\
\hline 'SLM046' & $4458 \mathrm{a}-\mathrm{i}$ & 3823 h-p & 0.83 & 635 & 4140.5 & 4128.3 & 0.89 & 1.06 & 0.86 & 14.24 \\
\hline 'Okapi' & 4300 b-k & $36001-p$ & 0.95 & 700 & 3950 & 3934.5 & 0.82 & 0.99 & 0.84 & 16.28 \\
\hline 'Orient' & 4114 e-n & 3329 opq & 1.11 & 785 & 3721.5 & 3700.7 & 0.72 & 0.92 & 0.81 & 19.08 \\
\hline 'Elvice' & $36001-p$ & $3500 \mathrm{n}-\mathrm{q}$ & 0.16 & 100 & 3550 & 3549.6 & 0.66 & 0.97 & 0.97 & 2.78 \\
\hline
\end{tabular}

Ys: yield of cultivar under stress, Yp: yield of cultivar under irrigated condition, SSI: stress susceptibility index, TOL: Tolerance, MP: mean productivity, GMP: Geometric mean productivity, STI: stress tolerance index, YI: yield index, YSI: yield stability index

Tab. 3. Resistance indices of 23 rapeseed genotypes under stress and non-stress environments for oil yield in 2008-2009

\begin{tabular}{|c|c|c|c|c|c|c|c|c|c|c|}
\hline Cultivar & Yp $\left(\mathrm{kg} \cdot \mathrm{ha}^{-1}\right)$ & Ys $\left(\mathrm{kg} \cdot \mathrm{ha}^{-1}\right)$ & SSI & TOL & MP & GMP & STI & YI & YSI & Reduction (\%) \\
\hline 'SW0756' & 1803 a-d & $1061 \mathrm{~h}$ & 1.18 & 742 & 1432 & 1383.1 & 0.59 & 0.9 & 0.59 & 41.15 \\
\hline 'Modena' & $2197 \mathrm{a}$ & $1176 \mathrm{gh}$ & 1.34 & 1021 & 1686.5 & 1607.4 & 0.79 & 1 & 0.54 & 46.47 \\
\hline 'Geronimo' & $2075 \mathrm{ab}$ & $1193 \mathrm{gh}$ & 1.22 & 882 & 1634 & 1573.4 & 0.76 & 1 & 0.57 & 42.51 \\
\hline 'Elite' & $1908 \mathrm{a}-\mathrm{d}$ & $1392 \mathrm{e}-\mathrm{h}$ & 0.78 & 516 & 1650 & 1629.7 & 0.82 & 1.2 & 0.73 & 27.04 \\
\hline 'Opera' & 1734 b-e & $997.9 \mathrm{~h}$ & 1.22 & 734.3 & 1366.85 & 1316.6 & 0.54 & 0.85 & 0.58 & 42.35 \\
\hline 'ARC-4' & $1631 \mathrm{c}-\mathrm{f}$ & $1009 \mathrm{~h}$ & 1.09 & 622 & 1320 & 1282.8 & 0.5 & 0.86 & 0.62 & 38.14 \\
\hline 'ARG-91004' & $1923 \mathrm{a}-\mathrm{d}$ & $1084 \mathrm{~h}$ & 1.26 & 839 & 1503.5 & 1443.8 & 0.64 & 0.92 & 0.56 & 43.63 \\
\hline 'ARC-5' & 1859 a-d & $1064 \mathrm{~h}$ & 1.23 & 795 & 1461.5 & 1406.4 & 0.61 & 0.91 & 0.57 & 42.76 \\
\hline 'ARC-2' & 1754 b-e & $1111 \mathrm{~h}$ & 1.05 & 643 & 1432.5 & 1395.9 & 0.6 & 0.95 & 0.63 & 36.66 \\
\hline 'Digger' & $1557 \mathrm{~d}-\mathrm{g}$ & $1071 \mathrm{~h}$ & 0.89 & 486 & 1314 & 1291.3 & 0.51 & 0.91 & 0.69 & 31.21 \\
\hline 'Adder' & 1849 a-d & $1079 \mathrm{~h}$ & 1.2 & 770 & 1464 & 1412.5 & 0.62 & 0.92 & 0.58 & 41.64 \\
\hline 'Milena' & $1841 \mathrm{a}-\mathrm{d}$ & $1105 \mathrm{~h}$ & 1.15 & 736 & 1473 & 1426.3 & 0.63 & 0.94 & 0.6 & 39.98 \\
\hline 'RG-9908' & $2030 \mathrm{abc}$ & $1159 \mathrm{gh}$ & 1.235 & 871 & 1594.5 & 1533.9 & 0.72 & 0.99 & 0.57 & 42.91 \\
\hline 'Dexter' & 1859 a-d & $1218 \mathrm{gh}$ & 0.99 & 641 & 1538.5 & 1504.7 & 0.7 & 1.04 & 0.65 & 34.48 \\
\hline 'Alice' & $1787 \mathrm{bcd}$ & $1212 \mathrm{gh}$ & 0.93 & 575 & 1499.5 & 1471.7 & 0.67 & 1.03 & 0.68 & 32.18 \\
\hline 'Olara' & $1790 \mathrm{bcd}$ & $1316 \mathrm{fgh}$ & 0.76 & 474 & 1553 & 1534.8 & 0.73 & 1.12 & 0.73 & 26.48 \\
\hline 'Ebonite' & $2105 \mathrm{ab}$ & 1386 e-h & 0.98 & 719 & 1745.5 & 1708.1 & 0.9 & 1.18 & 0.66 & 34.16 \\
\hline 'Syn-4' & $1848 \mathrm{a}-\mathrm{d}$ & $1371 \mathrm{e}-\mathrm{h}$ & 0.74 & 477 & 1609.5 & 1591.7 & 0.78 & 1.17 & 0.74 & 25.81 \\
\hline 'Zarfam' & $1518 \mathrm{~d}-\mathrm{g}$ & $1239 \mathrm{fgh}$ & 0.53 & 279 & 1378.5 & 1371.4 & 0.58 & 1.05 & 0.82 & 18.38 \\
\hline 'SLM046' & $1853 \mathrm{a}-\mathrm{d}$ & $1181 \mathrm{gh}$ & 1.04 & 672 & 1517 & 1479.3 & 0.68 & 1.01 & 0.64 & 36.26 \\
\hline 'Okapi' & $1560 \mathrm{~d}-\mathrm{g}$ & $1259 \mathrm{fgh}$ & 0.55 & 301 & 1409.5 & 1401.4 & 0.61 & 1.07 & 0.81 & 19.29 \\
\hline 'Orient' & $1529 \mathrm{~d}-\mathrm{g}$ & $1097 \mathrm{~h}$ & 0.81 & 432 & 1313 & 1295.1 & 0.52 & 0.93 & 0.72 & 28.25 \\
\hline 'Elvice' & $1340 \mathrm{fgh}$ & $1208 \mathrm{gh}$ & 0.28 & 132 & 1274 & 1272.3 & 0.5 & 1.03 & 0.9 & 9.85 \\
\hline
\end{tabular}

Ys: yield of cultivar under stress, Yp: yield of cultivar under irrigated condition, SSI: stress susceptibility index, TOL: Tolerance, MP: mean productivity, GMP: Geometric mean productivity, STI: stress tolerance index, YI: yield index, YSI: yield stability index 
Tab. 4. Resistance indices of 23 rapeseed genotypes under stress and non-stress environments for oil yield in 2009-2010

\begin{tabular}{|c|c|c|c|c|c|c|c|c|c|c|}
\hline Cultivar & Yp $\left(\mathrm{kg} \cdot \mathrm{ha}^{-1}\right)$ & Ys (kg.ha $\left.{ }^{-1}\right)$ & SSI & TOL & MP & GMP & STI & $\mathrm{YI}$ & YSI & Reduction(\%) \\
\hline 'SW0756' & 1728 g-o & 1475 nop & 0.89 & 253 & 1601.5 & 1596.5 & 0.68 & 0.92 & 0.85 & 14.64 \\
\hline 'Modena' & $2110 a-e$ & $1850 \mathrm{~d}-\mathrm{m}$ & 0.75 & 260 & 1980 & 1975.7 & 1.05 & 1.15 & 0.88 & 12.32 \\
\hline 'Geronimo' & $2001 \mathrm{a}-\mathrm{h}$ & $1823 \mathrm{~d}-\mathrm{m}$ & 0.54 & 178 & 1912 & 1909.9 & 0.98 & 1.13 & 0.91 & 8.89 \\
\hline 'Elite' & $2245 \mathrm{a}$ & $1857 \mathrm{c}-\mathrm{m}$ & 1.05 & 388 & 2051 & 2041.8 & 1.12 & 1.15 & 0.83 & 17.28 \\
\hline 'Opera' & $1912 \mathrm{a}-\mathrm{l}$ & $1308 \mathrm{pq}$ & 1.93 & 604 & 1610 & 1581.4 & 0.67 & 0.81 & 0.68 & 31.59 \\
\hline 'ARC-4' & $1960 a-i$ & 1641 i-p & 0.99 & 319 & 1800.5 & 1793.4 & 0.86 & 1.02 & 0.84 & 16.27 \\
\hline 'ARG-91004' & $1888 \mathrm{~b}-\mathrm{l}$ & $1729 \mathrm{~g}-\mathrm{o}$ & 0.51 & 159 & 1808.5 & 1806.7 & 0.88 & 1.07 & 0.91 & 8.42 \\
\hline 'ARC-5' & $2149 \mathrm{a}-\mathrm{d}$ & $1873 \mathrm{c}-1$ & 0.78 & 276 & 2011 & 2006.3 & 1.08 & 1.16 & 0.87 & 12.84 \\
\hline 'ARC-2' & $1849 \mathrm{~d}-\mathrm{m}$ & $1528 \mathrm{~m}-\mathrm{p}$ & 1.06 & 321 & 1688.5 & 1680.8 & 0.76 & 0.95 & 0.87 & 17.36 \\
\hline 'Digger' & $1925 \mathrm{a}-\mathrm{k}$ & $1585 \mathrm{k}-\mathrm{p}$ & 1.08 & 340 & 1755 & 1746.7 & 0.82 & 0.98 & 0.82 & 17.66 \\
\hline 'Adder' & $15751-p$ & 1479 nop & 0.37 & 96 & 1527 & 1526.2 & 0.62 & 0.92 & 0.94 & 6.09 \\
\hline 'Milena' & $2044 \mathrm{a}-\mathrm{g}$ & $1712 \mathrm{~g}-\mathrm{o}$ & 1.69 & 565 & 1761.5 & 1738.7 & 0.81 & 0.92 & 0.72 & 27.64 \\
\hline 'RG-9908' & $2191 \mathrm{abc}$ & 1465 nop & 2.02 & 726 & 1828 & 1791.6 & 0.86 & 0.91 & 0.67 & 33.13 \\
\hline 'Dexter' & $2044 \mathrm{a}-\mathrm{g}$ & $1944 a-j$ & 0.29 & 100 & 1994 & 1993.4 & 1.07 & 1.21 & 0.95 & 4.89 \\
\hline 'Alice' & 1472 nop & $1066 \mathrm{q}$ & 1.68 & 406 & 1269 & 1252.6 & 0.42 & 0.66 & 0.72 & 27.58 \\
\hline 'Olara' & $1913 \mathrm{a}-1$ & $1613 j-p$ & 0.96 & 300 & 1763 & 1756.6 & 0.83 & 1 & 0.84 & 15.68 \\
\hline 'Ebonite' & 2077 a-f & $1614 j-p$ & 1.36 & 463 & 1845.5 & 1830.9 & 0.9 & 1 & 0.78 & 22.29 \\
\hline 'Syn-4' & $2218 \mathrm{ab}$ & 1783 e-n & 1.19 & 435 & 2000.5 & 1988.6 & 1.06 & 1.11 & 0.8 & 19.61 \\
\hline 'Zarfam' & $1745 \mathrm{f}-\mathrm{o}$ & $1634 \mathrm{i}-\mathrm{p}$ & 0.39 & 111 & 1689.5 & 1688.6 & 0.77 & 1.01 & 0.94 & 6.36 \\
\hline 'SLM046' & $1951 a-j$ & 1697 h-o & 0.79 & 254 & 1824 & 1819.6 & 0.89 & 1.05 & 0.87 & 13.02 \\
\hline 'Okapi' & $1849 \mathrm{~d}-\mathrm{m}$ & 1628 i-p & 0.73 & 221 & 1738.5 & 1734.9 & 0.81 & 1.01 & 0.88 & 11.95 \\
\hline 'Orient' & $1876 \mathrm{c}-1$ & 1428 op & 1.46 & 448 & 1652 & 1636.7 & 0.72 & 0.88 & 0.76 & 23.88 \\
\hline 'Elvice' & $1621 \mathrm{i}-\mathrm{p}$ & 1577 l-p & 0.16 & 44 & 1599 & 1598.8 & 0.69 & 0.98 & 0.97 & 2.71 \\
\hline
\end{tabular}

Ys: yield of cultivar under stress, Yp: yield of cultivar under irrigated condition, SSI: stress susceptibilityindex, TOL: Tolerance, MP: mean productivity, GMP: Geometric mean productivity, STI: stress tolerance index, YI: yield index, YSI: yield stability index

Tab. 5. Simple correlation coefficients of stress indices with seed yield of 23 rapeseed cultivars

\begin{tabular}{|c|c|c|c|c|c|c|c|c|c|}
\hline & Ys $\left(\mathrm{kg} \cdot \mathrm{ha}^{-1}\right)$ & SSI & TOL & MP & GMP & STI & YI & YSI & Reduction (\%) \\
\hline Yp & $0.54^{* *}$ & $0.45^{*}$ & $0.91^{* *}$ & $0.66^{* *}$ & $0.88^{* *}$ & $0.87^{* *}$ & $0.51^{*}$ & $-0.49^{*}$ & $0.49^{*}$ \\
\hline Ys & 1 & $-0.5^{*}$ & $0.24 \mathrm{~ns}$ & $-0.269 \mathrm{~ns}$ & $0.87^{* *}$ & $0.88^{* *}$ & $0.99^{* *}$ & $0.45^{*}$ & $-0.45^{*}$ \\
\hline SSI & & 1 & $0.03 \mathrm{~ns}$ & $0.94^{* *}$ & $-0.02 \mathrm{~ns}$ & $-0.03 n s$ & $-0.49^{*}$ & $-0.98^{* *}$ & $0.98^{* *}$ \\
\hline TOL & & & 1 & $0.29 \mathrm{~ns}$ & $0.99^{* *}$ & $0.99^{* *}$ & $0.82^{* *}$ & $-0.09 \mathrm{~ns}$ & $0.09 \mathrm{~ns}$ \\
\hline MP & & & & 1 & $0.22 \mathrm{~ns}$ & $0.22 \mathrm{~ns}$ & $-0.3 n s$ & $-0.97^{* *}$ & $0.97^{* *}$ \\
\hline GMP & & & & & 1 & $0.99^{* *}$ & $0.86^{* *}$ & $-0.03 \mathrm{~ns}$ & $0.03 \mathrm{~ns}$ \\
\hline STI & & & & & & 1 & $0.86^{* *}$ & $-0.02 \mathrm{~ns}$ & $0.02 \mathrm{~ns}$ \\
\hline YI & & & & & & & 1 & $0.47^{*}$ & $-0.47^{*}$ \\
\hline YSI & & & & & & & & 1 & $-1^{* *}$ \\
\hline
\end{tabular}

Ys: yield of cultivar under stress, Yp: yield of cultivar under irrigated condition, SSI: stress susceptibility index, TOL: Tolerance, MP: mean productivity, GMP: Geometric mean productivity, STI: stress tolerance index, YI: yield index, YSI: yield stability index. Ns: not significant; ${ }^{*} P<0.05 ;{ }^{* *} P<0.01$

The greater value of SSI indicate the larger drought tolerance under stress and the cultivars with greater SSI are higher drought sensitivity (Tab. 1, 2,3 and 4). A positive correlation between SSI and irrigated yield (Yp) and a negative correlation between SSI and yield under stress (Ys) (Tab. 5) suggest that selection based on SSI will result in increased yield under well-watered conditions. 'Alice', 'Orient' and 'Elvice', for example, with relatively low yields under stress conditions, exhibited high MP values (Tab. 1 and 2, Fig. 1). The MP can be related to yield under stress only when stress is not too severe and the difference between yield under stress and nonstress conditions is not too big (Sio-Se Mardeh et al., 2006). Hossain et al. (1990) used MP as a resistance criterion for wheat cultivars in moderate stress conditions. Ahmad Zadeh (1997) introduced MP as appropriate criterion for selection of high yield and drought tolerance in corn. The 'Modena', 'Opera', 'ARC-4', 'ARG-91004', 'ARC-5', 'ARC-2', 'Milena', 'RG9908' and 'Ebonite' with high yield under stress produced a lower yield under non-stress conditions and showed the lowest SSI (Tab. 1 and 2). SSI showed a negative correlation with yield under stress (Tab. 5).

No significant correlation was found between yield under stress and MP in various stress stages (Tab. 5), 
showing that MP will not discriminate drought sensitive cultivars under such conditions. SSI has been widely used by researchers to identify sensitive and resistant genotypes (Clarke et al., 1984, Golabadi et al., 2006; Sio-Se Mardeh et al., 2006).

In the present study, the means of GMP and STI appeared to be a suitable selection index to distinguish resistant cultivars (Tab. 5). In the second year, 'Modena', 'Elite,' 'Olara,' 'Ebonite' and 'Syn- 4 ' with a highest GMP and STI were identified as resistant cultivars, whereas 'Opera', 'ARC-4', 'Digger', 'Zarfam,' 'Orient' and 'Elvice', with the lower GMP and STI were sensitive (Tab. 1) but in the second year, 'Modena,' 'Geronimo,' 'Elite,' 'ARC-5', 'Dexter' and 'Syn-4' cultivars also had highest GMP and STI (Tab. 2). The difference between the highest and lowest yielding cultivars was about 1565 and $868 \mathrm{~kg} \mathrm{ha}^{-1}$ in 2008 2009 and 1,700 and $1,977 \mathrm{~kg} \mathrm{ha}^{-1}$ in 2009-2010 in nonstress and stress conditions, respectively (Tab. 1 and 2). YI, proposed by Gavuzzi et al. (1997), was significantly correlated with stress yield. This index ranks cultivars only on the basis of their yield under stress (Tab. 5) and so does not discriminate genotypes of group A. YSI, as Bouslama and Schapaugh (1984) stated, evaluates the yield under stress of a cultivar relative to its non-stress yield, and should be an indicator of drought resistant genetic material. As a result, the cultivars with a high YSI are expected to have high yield under both stress and non-stress conditions (Shirani Rad and Abbasian, 2011). In the present study, however, cultivars with the highest YSI exhibited the least yield under non-stress conditions and the highest yield under stress conditions (Tab. 1 and 2).
The results indicated that there was a positive and significant correlation among Ys with YI, STI, GMP and they hence were better predictors of Yp and Ys than TOL, SSI and YSI (Tab. 5). Farshadfar et al. (2001) believed that most appropriate index for selecting stress-tolerant cultivars is index which has partly high correlation with seed yield under stress and non-stress conditions. The observed relations were consistent with those reported by Fernandez (1992) in mungbean, Farshadfar and Sutka (2002) in maize and Golabadi et al. (2006) in durum wheat. The results of calculated seed from indirect selection in moisture stress environment would improve yield in moisture stress environment better than selection from non-moisture stress environment. Wheat breeders should, therefore, take into account the stress severity of the environment when choosing an index. STI, GMP and YI were able to identify cultivars producing high yield in both conditions. It is concluded that the effectiveness of selection indices depends on the stress severity supporting the idea that only under moderate stress conditions, potential yield greatly influences yield under stress (Blum, 1996; Panthuwan et al., 2002).

Content of oil yield has the highest importance in production profitability (Robertson and Holland, 2004). Since oil yield was obtained through multiplying oil content by seed yield and also magnitude of changing oil content in modified rapeseed cultivars is low, therefore seed yield has the greatest effect on oil yield. Through breeding and selecting of cultivars for achieving high seed yield, high oil yield can also be achieved. Also, seed yield and oil yield compared to 1000-seed weight and oil content are

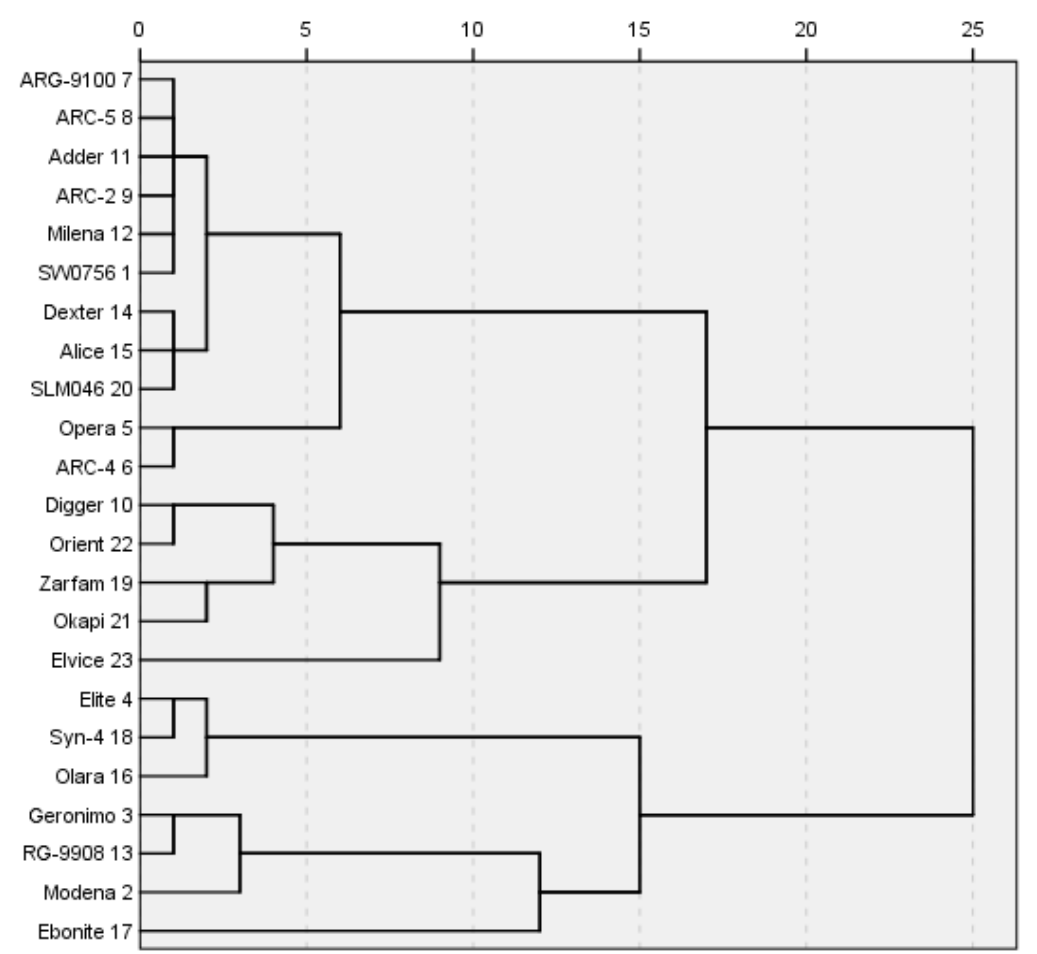

Fig. 2. Dendrogram resulting from cluster analysis of genotypes based on stress tolerance and susceptibility indices for grain yield in normal and stress condition 
170

more affected by environmental conditions (Khoshnazar Parshokohi et al., 2000). The expression of oil yield, as one of the most important rapeseed quantitative traits, is greatly influenced not only by genotype, but also by environment and complex genotype $\times$ environment interactions (Habekotte, 1997; Sidlauskas and Bernotas, 2003). Therefore, it is always attempted to test the stability or consistency of each genotype in wide range of different environments. The obtained data is very useful in selection of the best genotypes, making this kind of research quite important for breeders and growers alike (Tuck et al., 2006). Beside high stability for oil yield, these genotypes also may be considered as wide adaptable for the most important trait of rapeseed and recommended for planting in different environments (Marjanović-Jeromela et al., 2008). Evaluation of indices of YI, GMP and STI for oil yield in different irrigation showed that 'Modena', 'Geronimo,' 'Elite' and 'Ebonite' cultivars have the greatest tolerance in the first year but in the second year, 'Modena', 'Geronimo,' 'Elite', 'ARC-5', 'Dexter' and 'Syn-4' cultivars have the highest tolerance. Also, in TOL and SSI indices, 'Elvice' and 'Zarfam' cultivars had least numeral value and the highest tolerance (Tab. 3 and 4).

Cluster analysis has been widely used for description of genetic diversity and grouping based on similar characteristics (Golestani et al., 2007; Golabadi et al., 2006; Malek Shahi et al., 2009; Souri et al., 2005). As it appears in Fig. 2 , the genotypes were classified in three groups with low intra- and high extra-group similarities.

\section{Conclusions}

This success has largely been achieved through fieldbased empirical selection for stress tolerance. YI, GMP and STI indices which highly correlated with seed yield in both environments are introduced as the best indices. They are suitable to screen drought-tolerant, high yielding genotypes (e.g. 'Modena', 'Geronimo', 'Elite', 'Syn-4' and 'SLM046') in both non stress and drought stress environments. These results relatively agreed with Shirani Rad and Abbasian (2011) and Mohammadi et al. (2011) that aforementioned indices for having positive and significant correlation with seed yield of rapeseed cultivars at drought stress and non-stress conditions and these indices were an appropriate criterion for recognition of high yield and drought tolerance genotypes.

\section{References}

Afridi MZ, Tariq M, Shood A (2002). Some aspects of NPK nutrition for improved yield and oil contents of canola. Asian J Plant Sci 5:507-509.

Ahmad Zadeh A (1997). Definition of the best drought tolerant index in corn selective lines. M.Sc. thesis. College of Agriculture, University of Tehran, Iran, 238 p. (in Farsi).

Ahmadi M, Bahrani MJ (2009). Yield and yield components of rapeseed as influenced by water stress at different growth stages and nitrogen levels. Am-Euras J Agricult Environ Sci 5:755-761.

Blum A (1996). Crop responses to drought and the interpretation of adaptation. Plant Growth Regul 20:135-148.

Bouslama M, Schapaugh WT (1984). Stress tolerance in soybean. Part 1. Evaluation of three screening techniques for heat and drought tolerance. Crop Sci 24:933-937.

Bruckner PL, Frohberg RC (1987). Rate and duration of grain fill in spring wheat. Crop Sci 27:451-455.

Choukan R, Taherkhani T, Ghannadha MR, Khodarahmi $M$ (2006). Evaluation of drought tolerance in grain maize inbred lines using drought tolerance indices. Iran J Agricult Sci 8:79-89 (in Farsi).

Clarke JM, de Pauw RM, Townley-Smith TM (1992). Evaluation of methods for quantification of drought tolerance in wheat. Crop Sci 32:728-732.

Clarke JM, Towenley-Smith TM, McCaig TN, Green DG (1984). Growth analysis of spring wheat cultivars of varying drought resistance. Crop Sci 24:537-541.

Dehdari A (2003). Genetic analysis of salt tolerance in wheat crosses. Ph.D. thesis. Isfahan University of Technology, Isfahan, Iran, 141 p. (in Farsi).

Farshadfar EA, Sutka J (2002). Multivariate analysis of drought tolerance in wheat substitution lines. Cereal Res Communic 31:33-39.

Farshadfar EA, Zamani MR, Matlabi M, Emam-Jome EE (2001). Selection for drought resistance chickpea lines. Iran J Agricult Sci 32:65-77 (in Farsi).

Fernandez GCJ (1992). Effective selection criteria for assessing stress tolerance. Proceedings of the International Symposium on Adaptation of Vegetables and Other Food Crops in Temperature and Water Stress Tolerance. Asian Vegetable Research and Development Centre, Taiwan, 257-270 p.

Fischer RA, Maurer R (1978). Drought resistance in spring wheat cultivars. I. Grain yield response. Austr J Agricult Res 29:897-907.

Gavuzzi P, Rizza F, Palumbo M, Campanile RG, Ricciardi GL, Borghi B (1997). Evaluation of field and laboratory predictors of drought and heat tolerance in winter cereals. Can J Plant Sci 77:523-531.

Golabadi M, Arzani A, Maibody SAM (2006). Assessment of drought tolerance in segregating populations in durum wheat. Afr J Agricult Res 5:162-171.

Golestani M, Pakniat H (2007). Evaluation of drought tolerance indices in sesame lines. J Sci Technol Agricult Natural Res 41:141-149.

Habekotte B (1997). Identification of strong and weak yield determining components of winter oilseed rape compared with winter wheat. Europ J Agronom 7:315-321.

Hossain ABS, Sears AG, Cox TS, Paulsen GM (1990). Desiccation tolerance and its relationship to assimilate partitioning in winter wheat. Crop Sci 30:622-627. 
Khayatnezhad M, Gholamin R, Jamaati-e-Somarin S, Zabihie-Mahmoodabad R (2010). Study of drought tolerance of maize genotypes using the stress tolerance index. Am-Eur J Agricult Environ Sci 9:359-363.

Khoshnazar Parshokohi R, Ahmadi MR, Ghanadha MR (2000). Investigation of adaptation and yield comparison in lines and rapeseed cultivars. Iran JAgricult Sci 31:341-351 (in Farsi).

Malek Shahi F, Dehghani H, Alizadeh B (2009). Study of drought tolerance indices in some cultivars of winter rapeseed (Brassica napus L.). J Sci Technol Agricult Natural Res 48:78-89.

Marjanovic-Jeromela A, Marinkovic R, Mijic A, Jankulovska M, Zdunic Z, Nagl N (2008). Oil Yield Stability of Winter Rapeseed (Brassica napus L.) Genotypes. Agricult Conspec Sci 73(4):217-220.

Mendham NJ, Salisbury PA (1995). Physiology, crop development, growth and yield, 11-64 p. In: Kimber DS, McGregor DI (Eds.). Brassica Oilseeds: Production and Utilization. CAB International, London.

Mitra J (2001). Genetics and genetic improvement of drought resistance in crop plants. Current Sci 80:758-762

Mohammadi M, Karimizadeh F, Abdipour M (2011). Evaluation of drought tolerance in bread wheat genotypes under dryland and supplemental irrigation conditions. Austr J Crop Sci 5:487-493.

Panthuwan G, Fokai S, Cooper M, Rajatasereekul S, O’Toole JC (2002). Yield response of rice genotypes to different types of drought under rainfed lowlands. Part 1: grain yield and yield components. Field Crops Res 41:45-54.

Richards RA, Rebetzke GJ, Condon AG, Herwaarden AF (2002) Breeding opportunities for increasing the efficiency of water use and crop yield in temperate cereals. Crop Sci 42:111-121
Robertson MJ, Holland JF (2004). Production risk of canola in the semi-arid subtropics of Australia. Austr J Agricult Res 55:525-538

Rosielle AA, Hamblin J (1981). Theoretical aspects of selection for yield in stress and non-stress environment. Crop Sci 21:943-946.

SAS System for Windows (1996). Release 8.02. SAS Institute Inc. Cary, USA.

Shirani Rad AH, Abbasian A (2011). Evaluation of drought tolerance in winter rapeseed cultivars based on tolerance and sensitivity indices. Žemdirbystė Agricult 98:41-48.

Sidlauskas G, Bernotas S (2003). Some factors affecting seed yield of spring oilseed rape (Brassica napus L.). Agronom Res 1:229-243.

Sio-Se Mardeh A, Ahmadi A, Poustini K, Mohammadi V (2006). Evaluation of drought resistance indices under various environmental conditions. Field Crops Res 98:222229.

Souri J, Dehghani H, Sabbaghzadeh SH (2005). Study of chickpea genotypes under water stress. Iran J Agricult Sci 36:1517-1527 (in Farsi).

Talebi R (2009). Effective selection criteria for assessing drought stress tolerance in durum wheat (Triticum durum Desf.). Gen Appl Plant Physiol 35:64-74.

Tuck G, Glendining M, Smith P, House J, Waltenbach M (2006). The potential distribution of bioenergy crops in Europe under present and future climate. Biomass Bioener 30:183-197.

Yousefi M (2004). Evaluation of selection efficiency for drought tolerant in wheat. M.Sc. Thesis. Isfahan University of Technology, Isfahan, Iran, 109 p. (in Farsi). 